OPEN ACCESS

Edited by:

Pietro Vajro,

University of Salerno, Italy

Reviewed by:

Nicola Santoro,

Yale University, United States

Claudia Mandato,

AORN Santobono-Pausilipon, Italy

*Correspondence:

Lucia Pacifico

lucia.pacifico@uniroma1.it

Specialty section:

This article was submitted to

Pediatric Gastroenterology,

Hepatology and Nutrition,

a section of the journa

Frontiers in Pediatrics

Received: 31 January 2020

Accepted: 20 March 2020

Published: 15 April 2020

Citation:

Pacifico L, Perla FM, Andreoli G, Grieco R, Pierimarchi $P$ and Chiesa $C$ (2020) Nonalcoholic Fatty Liver Disease Is Associated With Low Skeletal Muscle Mass in Overweight/Obese Youths. Front. Pediatr. 8:158

doi: 10.3389/fped.2020.00158

\section{Nonalcoholic Fatty Liver Disease Is Associated With Low Skeletal Muscle Mass in Overweight/Obese Youths}

\author{
Lucia Pacifico ${ }^{1 *}$, Francesco Massimo Perla ${ }^{1}$, Gianmarco Andreoli ${ }^{1}$, Rosangela Grieco ${ }^{1}$, \\ Pasquale Pierimarchi ${ }^{2}$ and Claudio Chiesa ${ }^{2}$ \\ ${ }^{1}$ Department of Pediatrics, Sapienza University of Rome, Rome, Italy, ${ }^{2}$ Institute of Translational Pharmacology, National \\ Research Council, Rome, Italy
}

Background: Recent studies in adult non-elderly and elderly individuals have reported a link between nonalcoholic fatty liver disease (NAFLD) and sarcopenia. Nonetheless, whether this relationship would be found outside these populations it is still unknown. Hence, we evaluated the relationship between NAFLD and skeletal muscle mass in children and adolescents with overweight/obesity.

Methods: Two-hundred and thirty-four overweight/obese youths were enrolled. NAFLD was diagnosed by ultrasononography, after exclusion of infectious and metabolic disorders. Forty of the patients with NAFLD had also liver biopsy. Total and regional lean body mass and total fat mass measurements were obtained by dual-energy $\mathrm{X}$-ray absorptiometry. The relative muscle mass (RMM) was defined as the percent of muscle mass $(\mathrm{kg})$ relative to the sum of muscle and fat $(\mathrm{kg})$ mass. Appendicular skeletal muscle mass (ASM) was calculated by the sum of muscle masses of the four limbs (kg), and expressed as percent of body weight.

Results: Subjects were stratified according to tertiles of RMM. The prevalence of abdominal obesity, dyslipidemia, insulin resistance, metabolic syndrome, NAFLD as well as biopsy-proven nonalcoholic steatohepatitis (NASH) was significantly increased in the lowest tertile of RMM. After controlling for age, sex and Tanner stage, children in the lowest tertile of RMM had an increased risk for NAFLD (OR=2.80, 95\% Cl=1.57-5.02) compared to those in the other two tertiles. This association persisted after additional adjustments for clinical and metabolic variables. Similarly, the risk of NAFLD in the lowest tertile of ASM/weight index was significantly higher compared to those in the other two tertiles after adjustment for the above confounders.

Conclusions: This is the first study to establish an independent association between low muscle mass and NAFLD/NASH in overweight/obese youths. Considering the worldwide increase of pediatric obesity, measurements of muscle mass may serve as useful method of identifying among obese children those at high metabolic risk who may need intensive lifestyle interventions to prevent NAFLD and its progression.

Keywords: NAFLD, NASH, youths, muscle mass, dual-energy X-ray absorptiometry 


\section{INTRODUCTION}

With the worldwide epidemic of obesity, nonalcoholic fatty liver disease (NAFLD) has emerged as the most prevalent chronic liver disease in adults as well as youths (1), and a rising indication for liver transplantation. NAFLD include a broad range of liver damage from simple steatosis, nonalcoholic steatohepatitis (NASH), to cirrhosis (2). Both adult and pediatric patients with NAFLD often manifest features of metabolic syndrome (MetS) (e.g., abdominal obesity, increased blood pressure, atherogenic dyslipidemia, insulin resistance, and glucose abnormalities), and thus are at greater risk for cardiovascular disease (CVD) (3-5). Although significant progresses in our knowledge of the pathophysiology of NAFLD have been achieved, mechanisms accounting for excess fat in the liver have not yet fully clarified. Therefore, the identification of all major factors affecting development of NAFLD earlier in life is crucial to prevent the progression of liver damage.

Recently, emerging evidence suggests that reduced skeletal muscle mass contributes to the risk of many chronic diseases including chronic liver diseases (6). The loss in lean body mass, that is sarcopenia, has long been associated with liver cirrhosis (7) as well as with a poor prognosis in patients with end-stage liver disease (8). According to the revised European consensus on definition and diagnosis of sarcopenia (9), this disease is characterized by low muscle quantity and quality. Infiltration of fat within and around skeletal muscle, that is myosteatosis, is directly related to age and adiposity, with an increased risk of adverse outcomes $(10,11)$. Although sarcopenia has long been thought to be a disease of the elderly (9), it has been recently reported to occur earlier in patients with cardiometabolic disorders such as obesity, diabetes mellitus, MetS and CVD (12). Indeed, several studies have reported in the adult nonelderly and elderly populations a link between sarcopenia and NAFLD (13), highlighting sarcopenia as an emerging risk factor for NAFLD and its progression. Nonetheless, whether this relationship would be found outside these populations it is still unknown. We here report the results of an observational, cross-sectional study investigating the relationship between NAFLD and skeletal muscle mass in a pediatric population with overweight/obesity.

\section{METHODS}

\section{Patients}

We enrolled 234 children and adolescents with overweight/obesity [body mass index $(\mathrm{BMI})>85$ th percentile according to age- and gender-specific percentiles of BMI] at the outpatient Clinics of the Department of Pediatrics, Sapienza University of Rome. Subjects were included if they were aged 6-18 years, nondiabetic, free from chronic diseases (including kidney, endocrinologic, and liver disorders) as well as from conditions known to influence body composition.

The study was approved by the Policlinico Umberto I Hospital Ethical Committee, and the parents of all participants gave informed consent.

\section{Clinical and Laboratory Data}

Anthropometric measurements were obtained with standard methods. Weight and Height were determined using an electronic scale and a wall-mounted stadiometer, respectively. BMI was calculated as body weight in kg divided by the height in meters squared. The pubertal status was evaluated by the Tanner stage. The degree of obesity was quantified using Cole's least mean-square method, which expresses BMI as standard deviation score (SDS) (14).

After the subject fasted overnight, blood samples were collected for the determination of glucose, insulin, total cholesterol, high-density lipoprotein cholesterol (HDL-C), triglycerides (TG), alanine aminotransferase (ALT), and aspartate aminotransferase. Insulin resistance was assessed by the homeostasis model assessment of insulin resistance (HOMA-IR) (15).

\section{Ultrasound Examination of the Liver}

Liver ultrasonography was performed by a single operator blinded to clinical and laboratory data. Diagnosis of fatty liver was based upon liver echogenicity exceeding that of the renal cortex and spleen, attenuation of ultrasound wave, loss of definition of the diaphragm, and poor delineation of the intrahepatic architecture (16).

\section{Dual Energy X-ray Absorptiometry Scans}

Total and regional lean body mass $(\mathrm{kg})$ and total fat mass $(\mathrm{kg})$ were obtained by a total body scanner (Hologic QDR-4500W). The relative muscle mass (RMM) was defined as the percent of muscle mass relative to the sum of muscle and fat mass [e.g., $100 \mathrm{x}$ muscle mass $(\mathrm{kg}) /$ muscle mass $(\mathrm{kg})+$ fat mass $(\mathrm{kg})]$, a measure to estimate the contribution of relative muscle mass to body composition (17-19). Appendicular skeletal muscle mass (ASM) was calculated by the sum of muscle masses in the four limbs (kg), and expressed as percent of body weight [ASM/weight (kg) x 100]. We also calculated ASM after adjusting for height squared $\left(\mathrm{ASM} / \mathrm{ht}^{2}\right)(17-19)$.

\section{Liver Biopsy}

A subgroup of obese children with ultrasound-diagnosed NAFLD had also liver biopsy to assess either the presence of NASH, or other competing liver diseases. Percutaneous needle liver biopsy was performed as previously described (15). The histologic features of steatosis, lobular and portal inflammation, and hepatocyte ballooning, and fibrosis were scored according to the NAFLD Clinical Research Network criteria (20). Diagnosis of NASH was based on the presence of steatosis with necroinflammation and hepatocyte ballooning (21).

\section{Definitions}

NAFLD was defined as the presence of fat in the liver on ultrasound (US) in the absence of an alternate identifiable cause. High blood pressure (BP) was defined by $\mathrm{BP} \geq 95$ th percentile for age, sex, and height (22). High waist circumference (WC), high TG, and low HDL-C were defined using age- and gender-specific percentiles (23). Impaired fasting glucose was defined by a value of fasting glucose $\geq 5.6 \mathrm{mmol} / \mathrm{L}$. MetS diagnosis was based on the 
TABLE 1 | Characteristics of study population according to tertiles of RMMª

\begin{tabular}{|c|c|c|c|c|}
\hline & \multicolumn{3}{|c|}{ RMM } & \multirow[t]{2}{*}{$P$} \\
\hline & Tertile1 & Tertile II & Tertile III & \\
\hline Number of subjects & 78 & 75 & 81 & \\
\hline Age, years & $11.3(2.3)$ & $11.5(2.8)$ & $12.5(3.0)$ & 0.06 \\
\hline Male sex, $n(\%)$ & $38(48.7)$ & $33(44.0)$ & $61(75.3)$ & 0.001 \\
\hline Prepubertal status, $n$ (\%) & 15 (19.2) & $16(21.3)$ & $14(17.2)$ & 0.26 \\
\hline Weight, kg & $65.0(21.0)$ & $59.3(22.1)$ & $64.1(21.1)$ & 0.21 \\
\hline Height, cm & $149.7(14.4)$ & $151.7(17.4)$ & $158.9(18.0)$ & 0.01 \\
\hline $\mathrm{BMI}\left(\mathrm{kg} / \mathrm{m}^{2}\right)$ & $28.3(4.5)$ & $24.7(3.7)$ & $24.6(3.3)$ & $<0.0001$ \\
\hline BMI-SD score & $2.13(0.40)$ & $1.70(0.39)$ & $1.60(0.39)$ & $<0.0001$ \\
\hline Waist circumference, $\mathrm{cm}$ & $91.2(13.2)$ & $84.8(14.0)$ & $84.6(12.0)$ & 0.002 \\
\hline Systolic BP, mmHg & $111(11)$ & $111(9)$ & $112(13)$ & 0.81 \\
\hline Diastolic BP, mmHg & $69(9)$ & $68(8)$ & $69(9)$ & 0.82 \\
\hline Total cholesterol, mg/dL & $170(39)$ & $177(50)$ & $163(41)$ & 0.19 \\
\hline $\mathrm{HDL}-\mathrm{C}, \mathrm{mg} / \mathrm{dL}$ & $46(13)$ & $47(12)$ & $49(10)$ & 0.17 \\
\hline Triglycerides, mg/dL & $97(72-141)$ & $91(65-128)$ & $76(52-123)$ & 0.031 \\
\hline TG/HDL-C ratio & $2.1(1.3-3.6)$ & $1.9(1.2-3.3)$ & $1.6(0.9-2.8)$ & 0.029 \\
\hline AST, U/L & $25(20-34)$ & 25 (20-30) & 23 (19-29) & 0.22 \\
\hline ALT, U/L & $25(17-47)$ & $22(15-35)$ & $20(15-32)$ & 0.15 \\
\hline Glucose, mg/dL & $4.8(0.8)$ & $4.8(0.45)$ & $4.8(0.39)$ & 0.84 \\
\hline Insulin, $\mu \mathrm{U} / \mathrm{mL}$ & $18(12-24)$ & $14(9-19)$ & $13(9-18)$ & 0.004 \\
\hline HOMA-IR & $3.7(2.5-4.8)$ & $2.8(1.9-3.9)$ & $2.8(2.0-4.0)$ & 0.015 \\
\hline Total body fat mass, kg & $28.0(10.0)$ & $22.8(9.0)$ & $19.0(5.9)$ & $<0.0001$ \\
\hline Total lean body mass, kg & $31.4(9.6)$ & $32.5(12.7)$ & $39.6(14.6)$ & $<0.0001$ \\
\hline RMM, \% & $53.0(3.3)$ & $58.9(1.5)$ & $66.9(4.8)$ & $<0.0001$ \\
\hline ASM, kg & $15.1(4.36)$ & $15.5(7.0)$ & $20.7(7.94)$ & $<0.0001$ \\
\hline ASM/weight index, \% & $24.5(1.73)$ & $26.2(2.86)$ & $30.8(7.42)$ & $<0.0001$ \\
\hline ASM $/ h^{2}$ & $6.7(1.0)$ & $6.4(1.6)$ & $7.6(2.0)$ & 0.001 \\
\hline NAFLD, $n(\%)$ & $43(55.2)$ & 25 (33.3) & 27 (33.3) & 0.006 \\
\hline
\end{tabular}

$R M M$, relative muscle mass; BMI, body mass index; BMI-SDS, BMI-SD score; $B P$, blood pressure; TG, triglycerides; HDL-C, high-density lipoprotein cholesterol; AST, aspartate aminotransferase; ALT, alanine aminotransferase; HOMA-IR, homeostasis model assessment of insulin resistance; ASM, appendicular skeletal muscle mass.

a Tertile I, RMM: < 56.72; tertile II, RMM: 56.72-61.99; tertile III, RMM: > 61.99.

Results are expressed as $n(\%)$, mean (SD) or median (interquartile range).

presence of at least 3 risk factors: high WC, elevated BP, low HDL$\mathrm{C}$ levels, hypertriglyceridemia and glucose impairment. Insulin resistance was established on the basis of the $90^{\text {th }}$ percentile of HOMA-IR specific for age and gender in overweight/obese children (24).

\section{Statistical Analysis}

Data are expressed as n (\%), mean (SD), or median (interquartile range). Overweight/obese children, with and without NAFLD, were stratified into tertiles of the total skeletal muscle mass. Differences among groups in quantitative variables were evaluated by one-way analysis of variance or Kruskal-Wallis test, as appropriate. Proportions were compared by the chi-square test. Partial correlation and linear regression coefficients were used to evaluate the relationship between variables. In order to assess the risk of NAFLD in the first (lowest) tertile of RMM as well as in the first (lowest) tertile of ASM/weight index compared
TABLE 2 | Age-, gender, and pubertal status- adjusted linear regression coefficients between RMM and clinical variables.

\begin{tabular}{lcccc}
\hline & \multicolumn{1}{c}{ All cases } & & NAFLD \\
\cline { 2 - 2 } & B coefficients $\mathbf{( 9 5 \% ~ C l )}$ & & B coefficients $(95 \%$ CI) \\
\hline Waist circumference, cm & $-0.320(-0.387,-0.253)^{\S}$ & & $-0.327(-0.414,-0.240) \S$ \\
Systolic BP, mmHg & $-0.081(-0.167,0.006)$ & & $-0.175(-0.290,-0.060)^{\star}$ \\
Diastolic BP, mmHg & $-0.101(-0.199,-0.002)^{\star}$ & & $-0.107(-0.261,0.047)$ \\
ALT & $-0.035(-0.060,-0.009)^{+}$ & & $-0.032(-0.061,-0.002)^{\star}$ \\
HDL-C, mg/dL & $0.057(-0.13,0.127)$ & & $0.173(0.07,0.278)^{+}$ \\
Triglycerides, mg/dL & $-0.014(-0.25,-0.002)^{\star}$ & & $-0.015(-0.029,-0.001)^{\star}$ \\
TG/HDL-C ratio & $-0.454(-0.78,-0.12)^{+}$ & & $-0.500(-0.87,-0.13)^{+}$ \\
Glucose & $-0.307(-1.76,1.15)$ & & $-0.903(-2.54,0.74)$ \\
Insulin & $-0.136(-0.204,-0.067)^{\S}$ & $-0.125(-0.197,-0.052)^{+}$ \\
HOMA-IR & $-0.396(-0.632,-0.161)^{+}$ & $-0.346(-0.585,-0.108)^{+}$ \\
\hline
\end{tabular}

RMM, relative muscle mass; NAFLD, nonalcoholic fatty liver disease; $\mathrm{Cl}$, confidence interval; BP, blood pressure; $A L T$, alanine aminotransferase; TG, triglycerides; HDL$C$, high-density lipoprotein cholesterol; HOMA-IR, homeostasis model assessment of insulin resistance.

${ }^{\star} P<0.05 ;{ }^{+} P<0.01 ;{ }^{\S} P<0.0001$.

to the combined second and third tertiles, we performed multiple logistic regression analysis controlled for age, sex, Tanner stage, and clinical confounders. Since the prevalence of cardiometabolic risk factors in the first tertile was markedly different to that observed in the second and third tertiles, the latter two tertiles have been combined.

\section{RESULTS}

\section{Characteristics of Study Population}

Descriptive characteristics of participant samples according to tertiles of RMM are summarized in Table 1. The subjects in the lowest tertile of RMM had the greatest BMI, BMI-SDS, WC, total body fat mass as well as the highest TG/HDL-C ratio, insulin, and HOMA-IR values compared with those in the other two tertiles of RMM. In comparison with subjects in the lowest RMM tertile, those in the middle or highest RMM tertile were more likely to be taller, and to have greater total lean body mass, absolute ASM, ASM/weight index, and ASM/ht ${ }^{2}$. There was a near-significance difference in age across the tertiles of RMM. Moreover, reduced RMM was significantly associated with an increased prevalence of NAFLD $(P=0.006)$. Conversely, there were no significant differences in Tanner stage, BP, total cholesterol, liver enzymes and glucose.

\section{Relationship Between RMM and Cardiometabolic Risk Factors}

In all study children and adolescents, after controlling for age, sex, and Tanner stage, RMM were negatively correlated with WC, diastolic BP, ALT, TG, TG/HDL ratio, insulin, and HOMA-IR values (Table 2). When the analysis was limited to patients with NAFLD, RMM were significantly associated with WC, systolic BP, TG, HDL-C and TG/HDL-C ratio, ALT, insulin and HOMA-IR values. In the subjects without NAFLD, RMM was significantly 
TABLE 3 | Prevalence of metabolic syndrome and its individual components according to RMM tertiles among the study population.

\begin{tabular}{|c|c|c|c|c|}
\hline & \multicolumn{4}{|c|}{ RMM } \\
\hline & $\begin{array}{l}\text { Tertile I } \\
(n=78)\end{array}$ & $\begin{array}{l}\text { Tertile II } \\
(n=75)\end{array}$ & $\begin{array}{l}\text { Tertile III } \\
(n=81)\end{array}$ & $\begin{array}{c}P \text { for linear } \\
\text { trend }\end{array}$ \\
\hline $\begin{array}{l}\text { Central obesity, } \\
\%(95 \% \mathrm{Cl})\end{array}$ & $\begin{array}{c}62.8 \\
(52.1-73.5)\end{array}$ & $\begin{array}{c}34.6 \\
(23.8-45.4)\end{array}$ & $\begin{array}{c}13.6 \\
(6.1-21.1)\end{array}$ & $<0.0001$ \\
\hline $\begin{array}{l}\text { Elevated BP, } \\
\%(95 \% \mathrm{Cl})\end{array}$ & $\begin{array}{c}29.5 \\
(19.4-39.6)\end{array}$ & $\begin{array}{c}17.3 \\
(8.7-25.9)\end{array}$ & $\begin{array}{c}27.1 \\
(17.4-36.8)\end{array}$ & 0.75 \\
\hline $\begin{array}{l}\text { High TG, } \\
\%(95 \% \mathrm{Cl})\end{array}$ & $\begin{array}{c}35.9 \\
(25.3-46.5)\end{array}$ & $\begin{array}{c}21.3 \\
(12.0-30.6)\end{array}$ & $\begin{array}{c}21.0 \\
(12.1-29.9)\end{array}$ & 0.033 \\
\hline $\begin{array}{l}\text { Low HDL-C, } \\
\%(95 \% \mathrm{Cl})\end{array}$ & $\begin{array}{c}38.5 \\
(27.7-49.3)\end{array}$ & $\begin{array}{c}20.0 \\
(11.0-29.0)\end{array}$ & $\begin{array}{c}19.7 \\
(11.0-28.4)\end{array}$ & 0.008 \\
\hline $\begin{array}{l}\text { Glucose } \geq 5.6 \mathrm{mmol} / \mathrm{L} \\
\%(95 \% \mathrm{Cl})\end{array}$ & $\begin{array}{c}1.3 \\
(0.2-6.9)^{\star}\end{array}$ & $\begin{array}{c}4.0 \\
(1.4-11.1)^{\star}\end{array}$ & $\begin{array}{c}3.7 \\
(1.27-10.3)^{\star}\end{array}$ & 0.36 \\
\hline $\begin{array}{l}\text { Insulin resistance, } \\
\%(95 \% \mathrm{Cl})\end{array}$ & $\begin{array}{c}70.5 \\
(60.4-80.6)\end{array}$ & $\begin{array}{c}52.0 \\
(35.0-69.0)\end{array}$ & $\begin{array}{c}51.8 \\
(40.9-62.7)\end{array}$ & 0.023 \\
\hline $\begin{array}{l}\text { NAFLD, } \\
\%(95 \% \mathrm{Cl})\end{array}$ & $\begin{array}{c}55.2 \\
(32.9-70.5)\end{array}$ & $\begin{array}{c}33.3 \\
(22.6-44.0)\end{array}$ & $\begin{array}{c}33.3 \\
(23.1-43.5)\end{array}$ & 0.006 \\
\hline $\begin{array}{l}\text { Metabolic syndrome, } \\
\%(95 \% \mathrm{Cl})\end{array}$ & $\begin{array}{c}29.5 \\
(19.3-39.7)\end{array}$ & $\begin{array}{c}12.0 \\
(4.6-19.3)\end{array}$ & $\begin{array}{c}3.7 \\
(1.3-10.3)^{\star}\end{array}$ & $<0.0001$ \\
\hline
\end{tabular}

$R M M$, relative muscle mass; $C l$, confidence interval; $B P$, blood pressure; $T G$, triglycerides; $H D L-C$, high-density lipoprotein cholesterol; NAFLD, nonalcoholic fatty liver disease. ${ }^{*}$ For these percentages, the exact $95 \% \mathrm{Cl}$ was calculated using the Wilson method.

correlated only with WC [B coefficient, -0.311 (95\% CI, -0.419/0.204); $P<0.0001]$.

\section{Cardiometabolic Profile Across RMM Tertiles in the Study Population}

The prevalence of high WC, high TG, low HDL-C, insulin resistance, NAFLD and MetS was significantly increased in the lowest tertile of RMM (Table 3 ).

To evaluate the potential independent contribution of RMM on NAFLD, multiple logistic regression analyses were performed (Table 4A). After controlling for age, sex and Tanner stage, children in the lowest tertile of RMM had an increased risk for NAFLD (OR $=2.80,95 \% \mathrm{CI}=1.57-5.02)$ compared to those in the other two tertiles. This association persisted after adjusting for potential confounders such as central obesity, elevated BP, elevated TG, low HDL-C, and insulin resistance, although the strength of association was slightly attenuated. When MetS (as a single clinical entity) was entered in the regression model in addition to age, gender and Tanner stage, the association remained statistically significant $(\mathrm{OR}=2.20,95 \%$ $\mathrm{CI}=1.19-4.05)$.

We also evaluated the adjusted associations of ASM/weight index with NAFLD (Table 4B). In model 1, controlled for age, sex and Tanner stage, the risk of NAFLD (OR $=2.99,95 \%$ $\mathrm{CI}=1.41-6.31)$ in the lowest tertile of ASM/weight index was significantly higher compared to that in the other two tertiles. These results remained unchanged after additional adjustments (including central obesity, elevated BP, elevated TG, low HDL-C, and insulin resistance, or MetS).
TABLE 4A | Adjusted odds ratio $(95 \% \mathrm{Cl}$ ) of the lowest tertile of RMM for NAFLD.

\begin{tabular}{lccc}
\hline & \multicolumn{3}{c}{ RMM } \\
\cline { 2 - 4 } & I & II and III & P value \\
\hline Adjusted model 1 & $2.80(1.57-5.02)$ & 1.00 (referent) & 0.001 \\
Adjusted model 2 & $2.18(1.17-4.07)$ & 1.00 (referent) & 0.014 \\
Adjusted model 3 & $2.18(1.13-4.18)$ & 1.00 (referent) & 0.019 \\
Adjusted model 4 & $2.20(1.19-4.05)$ & 1.00 (referent) & 0.012 \\
\hline
\end{tabular}

TABLE 4B | Adjusted odds ratio $(95 \% \mathrm{Cl})$ of the lowest tertile of ASM/weight index for NAFLD.

\begin{tabular}{lccl}
\hline & \multicolumn{3}{c}{ ASM } \\
\cline { 2 - 4 } & \multicolumn{1}{c}{ I } & II and III & P value \\
\hline Adjusted model 1 & $2.99(1.41-6.31)$ & 1.00 (referent) & 0.004 \\
Adjusted model 2 & $2.29(1.04-5.06)$ & 1.00 (referent) & 0.04 \\
Adjusted model 3 & $2.33(1.01-5.40)$ & 1.00 (referent) & 0.048 \\
Adjusted model 4 & $2.54(1.16-5.58)$ & 1.00 (referent) & 0.02 \\
\hline
\end{tabular}

Model 1: adjusted for age, gender and pubertal status; Model 2: adjusted for age, gender, pubertal status, and central obesity; Model 3: adjusted for age, gender, pubertal status, central obesity, high blood pressure, elevated triglycerides, low high-density lipoprotein cholesterol, and insulin resistance; Model 4: adjusted for age, gender, pubertal status and MetS.

$\mathrm{Cl}$, confidence interval.

\section{Findings in Children With Biopsy-Proven NAFLD}

Twenty-four children (60.0\%) had definite-NASH, while $16(40.0 \%)$ had not-NASH. In comparison with children and adolescents with not-NASH, those with NASH showed significantly lower RMM [mean, 55.7 (SD, 6.0) vs. 63.4 (6.0) \%; $P<0.0001$ ] and lower ASM/weight index [mean, 25.6 (SD, 2.8) vs. 28.6 (2.9) $\%$; $P=0.006]$. Also, the prevalence of NASH was significantly increased in the lowest tertile of RMM [70.8 (95\% CI, 61.8-79.8) \% vs. $29.2(20.2-38.2) \% ; P<0.001]$ as well as in the lowest tertile of ASM/weight index [62.5 (95\% CI, 47.5-77.5) $\%$ vs. $37.5(22.5-52.5) \%$; $P<0.003$ ] compared to those in the other two tertiles, respectively.

Some degree of fibrosis was present in $72.5 \%$ of patients with histologically diagnosed NAFLD, of whom $30 \%$ showed stage 1 , $40 \%$ stage 2 and $2.5 \%$ stage 3 fibrosis. There were no significant differences in RMM and ASM/weight index between patients with fibrosis grade $\geq 2$ and those with fibrosis $\leq 1$.

\section{DISCUSSION}

To our knowledge, this is the first study to assess in a pediatric population the relationship of skeletal muscle mass with NAFLD. We demonstrated that (1) overweight/obese youths with lower muscle mass have a greater risk of NAFLD compared to those with higher muscle mass; (2) the inverse association between NAFLD and muscle mass in children and adolescents is independent from anthropometric and metabolic variables; and (3) overweight/obese youths with lower muscle mass have 
a greater prevalence of cardiometabolic risk factors (e.g., central obesity, dyslipidemia, and insulin resistance) as well as MetS. In the subgroup of obese patients with US-diagnosed NAFLD who underwent liver biopsy, we also demonstrated the association between reduced muscle mass and NASH.

Several cross-sectional studies in adult patients have showed that sarcopenia is associated with NAFLD, NASH and NAFLDassociated advanced fibrosis (25-29). Notably, in a nationally representative sample of both obese and non-obese Korean adult individuals, Lee et al. (25) provided robust evidence of an independent association of NAFLD with sarcopenia. They demonstrated that patients with sarcopenia had a higher risk of NAFLD independently of the status of obesity as well as of MetS compared with subjects with a preserved muscle mass. More recently, in a large, longitudinal population-based cohort study, Kim et al. confirmed this association and suggested a causal link (30). The authors tested the effects of RMM modifications over time on the occurrence of new NAFLD or the resolution of pre-existing NAFLD (30). Skeletal muscle mass index (SMI) as assessed at baseline was inversely related to incident NAFLD and positively related to the resolution of preexisting NAFLD. Furthermore, an increase in SMI over 1-year period had significant favorable effects either on the development of new NAFLD or the improvement of pre-existing NAFLD, even after controlling for glycometabolic variables and baseline SMI (30).

Although in 1984 Forbes (31) described for the first time a low muscle mass phenotype in obese children, since then very little attention has been paid to its metabolic implications in pediatrics. Only recently, children and adolescents with low muscle mass and strength have been shown to be at increased risk of developing metabolic dysfunction and CVD $(18,32-$ 35 ), as previously reported in the adult nonelderly and elderly populations (6). In a large sample of U.S. youth, aged 8-20 years, Kim et al. (18) demonstrated an inverse association between RMM and cardiometabolic risk factors. In addition, they showed that the odds of having an adverse level for all risk factors with the exception of diastolic BP gradually diminish as RMM increases. A more recent study involving 660 apparently healthy adolescents, showed that those with low muscle mass (into the first quartile) had higher cardiometabolic risk (higher values of BMI z-score, WC, systolic BP, diastolic BP, TG, TC/HDL-C, insulin, HOMA-IR, and MetS z-score) than adolescents in the other quartiles regardless of nutritional status (17). Furthermore, low muscle mass increased the obesity-related cardiometabolic risk. In the present study involving a Caucasian pediatric population with overweight/obesity, we confirmed the inverse relationship between low muscle mass and cardiometabolic risk factors, and we first showed an independent association between low muscle mass (total and appendicular) and NAFLD as well as NASH.

There is growing recognition that reduced lean tissue, notably skeletal muscle, can co-occur in the presence of obesity, the so-called sarcopenic obesity (12, 36-38). Clinical evaluation of muscle mass in the obese subjects, however, is a real pitfall, since a reduced skeletal muscle mass may be masked by the presence of excess fat (37). The concordance of these two conditions, e.g., low level of muscle mass and excess weight, is associated with worse clinical outcomes than is either condition alone (39-41); therefore, attempts to preserve muscle mass and to lessen the consequences of low muscle mass might be more fruitful if initiated at childhood than if initiated at adulthood. Accordingly, in obese children a systematic muscle mass assessment is needed to improve diagnosis and treatment of those presenting a disparity in muscle and fat stores at an early stage. As such, more and more attention is being paid to the reference technology as well as to the diagnostic criteria to assess muscle mass in different contexts and populations including obese children.

Currently, various techniques are available for estimating or measuring muscle mass. Unfortunately, no consensus has yet been reached on the best technique to estimate or measure it (42). Among them, computed tomography (CT) and magnetic resonance imaging (MRI) are ideal in terms of accuracy, but their routine use in many clinical settings is compromized by the high cost of instrumentation, concerns of radiation exposure (for CT), contraindications for scanning (for MRI), and limited access to equipment $(36-38,42)$. Thus, both techniques are not suitable for population screening, nor available at early asymptomatic stages of the disease (37). Unsurprisingly, use of these gold standard techniques to assess the muscle mass in the clinical pediatric setting has been limited to children with endstage organ failure and/or increased fat stores requiring imaging evaluation of the underlying chronic diseases while awaiting solid organ transplantation $(43,44)$.

A yet poorly explored element of sarcopenia has been quantification of fatty infiltration of muscle. However, evaluation of fat deposition in muscles is complex, far from clinical practice, especially in children and adolescents, and may be obtained only using imaging techniques or more invasive methods like muscle biopsy (45). CT and MRI are considered the gold standard for intramuscular and intermuscular fat evaluation, respectively, while other body composition methods including DEXA do not allow to evaluate muscle quality parameters. Accordingly, this limitation warrants further studies assessing in a selected group of pediatric patients the relevance of myosteatosis by imaging techniques in childhood "sarcopenic obesity."

Bearing all these considerations in mind, despite many limitations, DEXA has been proposed as the standard technique for assessing muscle mass and body composition in research and clinical practice because of its ease of use, relatively low cost, minimal radiation exposure, short scan time and accessibility (42).

In addition to the commonly reported appendicular lean-mass estimates (i.e., estimation of the muscle masses in the four limbs, representing about $75 \%$ of total body muscle mass) $(37,42)$, using DEXA we also measured the contribution of RMM to body composition. To this end, we followed the method proposed by Kim et al. (18) as variation of the measure initially introduced by Park et al. (46) to evaluate in adults the relationship between muscle mass and MetS. In children the muscle-to-fat ratio (MFR) has been established as the key indicator of low muscle mass with the potential to stratify the risk of complications $(19,47)$. 
Indeed, in obese youths, the presence of a low muscle mass in a combination with a high fat mass, which would result in low MRF, may act synergistically leading to a more severe cardiometabolic risk (19).

NAFLD diagnosis was based in the majority of participants on US examination after exclusion of infectious and metabolic disorders, while only a small sample size of children underwent liver biopsy, which is the current standard to define the presence and severity of NAFLD. Although US is the imaging modality most widely used for the noninvasive assessment of liver steatosis, due to its significant advantages such as being largely available, relatively non-expensive, and easy to use, it has low sensitivity, particularly in children who have lower degrees of steatosis (e.g., involving $<33 \%$ of hepatocytes). In addition, US is inaccurate for quantification of steatosis in youths. For these reasons, the recent guidelines from the NASPGHAN Expert Committee have recommended ALT as the best screening test for NAFLD in children (48). However, in a very recent study, two screening strategies were compared: the NASPGHAN strategy using an ALT cut-off of $>2 x$ the gender-specific upper limit of normal and the ESPGHAN strategy using elevated ALT $>45 \mathrm{IU} / \mathrm{L}$ and/or fatty liver on ultrasound. The study showed that by relying on ALT values alone to screen for NAFLD, suspected NAFLD might be missed in many children who are at risk to develop the disease (49). In our study, it is possible that some subjects with US-diagnosis of NAFLD (due to low sensitivity) were enrolled in the control groups. However, the possible inclusion of controls with NAFLD may have led to underestimation of the differences in the RMM between cases and controls rather than the opposite.

Although this study was not designed to clarify the pathogenic link between sarcopenia and NAFLD, we acknowledge that the two conditions have in common several pathophysiologic processes, especially insulin resistance, chronic inflammation, and decreased physical activity (50). Skeletal muscle is the most effective organ for whole-body insulin-mediated glucose disposal (51), and therefore is the key element for maintaining effective glucose homeostasis in many chronic diseases. As such, the loss of muscle mass reduces the quantity of the primary target for insulin, favoring glucose intolerance and gluconeogenesis, which are key to the pathogenesis of NAFLD. Interestingly, Petersen et al. (52) demonstrated that, after high carbohydrate meals, young insulin-resistant subjects showed a marked defect in muscle glycogen synthesis and diverted much more of their ingested energy into hepatic de novo lipogenesis, resulting in increased hepatic triglycerides synthesis, while young insulinsensitive individuals stored more of their ingested energy in liver and muscle glycogen. In our study, low muscle mass was related to NAFLD independently of insulin resistance. Thus, it is plausible that other mechanisms could be involved, including subclinical inflammation and enhanced oxidative stress. Subjects with sarcopenia have elevated concentrations of C-reactive protein and inflammatory cytokines, which may promote skeletal muscle catabolism $(28,53)$. Low-grade inflammation and oxidative stress may also have a relevant role for the occurrence of NAFLD and its progression. Moreover, skeletal muscle is regarded as an endocrine organ able to secrete a series of cytokines so-called myokines, which constitute a broad network among metabolic tissues and organs including the liver (54). Myokines produced and released by contracting skeletal muscles counteract systemic inflammation and modulate glucose and lipid metabolism (55). This might account for the wellknown favorable effects of physical activity toward the diseases associated with "the diseasome of physical inactivity" (55). Importantly, there is a growing body of evidence supporting the beneficial effects of exercise and of physical fitness per $s e$ in the pathogenesis of NAFLD (56). As a matter of fact, exercise-only interventions (e.g., without modification of the diet) have been shown to result in a significant reduction of the amount of intrahepatic fat, even in the absence of significant weight changes (57). The influence of physical inactivity on both obesity and muscle reduction in children is common sense. Both physical inactivity and sedentary behavior with consequent muscle disuse can lead to a substantial decrease in lean body mass, creating a vicious cycle causing both progressive inactivity and sarcopenia.

Nutrition represents a key factor in the prevention and treatment of both sarcopenia and obesity. Sarcopenia is associated with an inadequate nutritional intake, and nutritional interventions are essential to improve sarcopenia and the subsequent morbidity and mortality in chronic liver diseases (58, 59). In contrast, obesity is a result of an excess consumption of energy, resulting in an imbalance between the energy intake and energy expenditure. As such, nutritional strategies for sarcopenic obesity should target not only an optimal energy intake in order to decrease excess fat mass, but also an optimal nutrient intake in order to increase skeletal muscle mass (60). Recent studies have shown that a combination of a moderate weight loss diet with concurrent exercise (especially resistance exercise) may improve body composition and physical performance in subjects with sarcopenic obesity (61).

Gut dysbiosis might have a role in the development and progression of NAFLD; therefore, the manipulation of gut microbiota with probiotics might prove an effective treatment strategy, particularly in subjects who are noncompliant to lifestyle interventions (62). Few studies in children have yielded promising results; however, several aspects of probiotic beneficial action in NAFLD (e.g., type of strain and doses) still need further elucidation (62). Of note, recently researchers are focusing their interests on the possible involvement of gut microbiota in the pathophysiology of sarcopenia and physical frailty (63). Alterations in the gut microbiota composition could in fact promote chronic inflammation and anabolic resistance, ultimately conditioning reduced muscle mass, impaired muscle function and adverse clinical outcomes (63). Thus, the relationship between gut microbiota and sarcopenic obesity remains a very promising area of research for the future.

Some limitations of the present study should be considered: (a) the cross-sectional nature, that does not allow determination of cause-and-effect relationship; (b) the relatively small sample size of children with biopsy-confirmed diagnosis of NAFLD; (c) missing data on physical activity; and (d) lack of data on modifications in muscle strength and quality. 
Nevertheless, despite these limitations, this is the first study to establish a close and independent association between low muscle mass and NAFLD/NASH in overweight/obese youths. Considering the worldwide increase of pediatric obesity, measurements of muscle mass may serve as useful method of identifying among obese children those at high cardiometabolic risk who may need more detailed medical examinations, and lifestyle interventions including adequate nutritional support and intensive exercise prescriptions for prevention of NAFLD and its progression.

\section{DATA AVAILABILITY STATEMENT}

The datasets generated for this study are available on request to the corresponding author.

\section{REFERENCES}

1. Loomba R, Sanyal AJ. The global nAFLD epidemic. Nat Rev Gastroenterol Hepatol. (2013) 10:686-90 doi: 10.1038/nrgastro.2013.171

2. Rinella ME. Nonalcoholic fatty liver disease: a systematic review. JAMA. (2015) 313:2263-73. doi: 10.1001/jama.2015.5370

3. Cali AM, De Oliveira AM, Kim H, Chen S, Reyes-Mugica M, Escalera S, et al. Glucose dysregulation and hepatic steatosis in obese adolescents: is there a link? Hepatology. (2009) 49:1896-903. doi: 10.1002/hep.22858

4. D'Adamo E, Cali AM, Weiss R, Santoro N, Pierpont B, Northrup V, et al. Central role of fatty liver in the pathogenesis of insulin resistance in obese adolescents. Diabetes Care. (2010) 33:1817-22 doi: 10.2337/dc10-0284

5. Kotronen A, Yki-Järvinen H. Fatty liver: a novel component of the metabolic syndrome. Arterioscler Thromb Vasc Biol. (2008) 28:27-38 doi: 10.1161/ATVBAHA.107.147538

6. Hsu CS, Kao JH. Sarcopenia and chronic liver diseases. Expert Rev Gastroenterol Hepatol. (2018) 12:1229-44. doi: 10.1080/17474124.2018.1534586

7. Kim G, Kang SH, Kim MY, Baik SK. Prognostic value of sarcopenia in patients with liver cirrhosis: a systematic review and meta-analysis. PLoS ONE. (2017) 12:e0186990. doi: 10.1371/journal.pone.0186990

8. Bhanji RA, Carey EJ, Yang L, Watt KD. The long winding road to transplant: how sarcopenia and debility impact morbidity and mortality on the waitlist. Clin Gastroenterol Hepatol. (2017) 15:1492-97 doi: 10.1016/j.cgh.2017.04.004

9. Cruz-Jentoft AJ, Bahat G, Bauer J, Boirie $\mathrm{Y}$, Bruyère $\mathrm{O}$, Cederholm $\mathrm{T}$, et al. Writing group for the European working group on sarcopenia in older people 2 (EWGSOP2), and the extended group for EWGSOP2. Sarcopenia: revised European consensus on definition and diagnosis. Age Ageing. (2019) 48:16-31. doi: 10.1093/ageing/afy169

10. Zhao Q, Zmuda JM, Kuipers AL, Jonnalagadda P, Bunker CH, Patrick AL, et al. Greater skeletal muscle fat infiltration is associated with higher allcause mortality among men of African ancestry. Age Ageing. (2016) 45:529-34. doi: 10.1093/ageing/afw062

11. Miljkovic I, Kuipers AL, Cauley JA, Prasad T, Lee CG, Ensrud KE, et al. Osteoporotic fractures in men study group. Greater skeletal muscle fat infiltration is associated with higher all-cause and cardiovascular mortality in older men. J Gerontol A Biol Sci Med Sci. (2015) 70:1133-40. doi: 10.1093/gerona/glv027

12. Kalyani RR, Corriere M, Ferrucci L. Age-related and disease-related muscle loss: the effect of diabetes, obesity, and other diseases. Lancet Diabetes Endocrinol. (2014) 2:819-29 doi: 10.1016/S2213-8587(14)70034-8

13. De Fré CH, De Fré MA, Kwanten WJ, Op de Beeck BJ, Van Gaal LF, Francque SM. Sarcopenia in patients with non-alcoholic fatty liver disease: is it a clinically significant entity? Obes Rev. (2019) 20:353-63. doi: $10.1111 /$ obr.12776

14. Cole TJ, Bellizzi MC, Flegal KM, Dietz WH. Establishing a standard definition for child overweight and obesity worldwide:

\section{ETHICS STATEMENT}

The studies involving human participants were reviewed and approved by Policlinico Umberto I Hospital Ethics Committee, Sapienza University. Written informed consent to participate in this study was provided by the participants' legal guardian/next of kin.

\section{AUTHOR CONTRIBUTIONS}

LP, FP, and CC conceptualized and designed the study. LP, FP, and RG collected the data. LP, PP, and CC analyzed and interpreted the data. LP and CC wrote the manuscript and all authors participated to the discussion of results and critically commented the manuscript for the final approval.

international survey. BMJ. (2000) 320:1240-3. doi: 10.1136/bmj.320. 7244.1240

15. Pacifico L, Di Martino M, Catalano C, Panebianco V, Bezzi M, Anania C, et al. T1-weighted dual-echo MRI for fat quantification in pediatric nonalcoholic fatty liver disease. World J Gastroenterol. (2011) 17:3012-9. doi: 10.3748/wjg.v17.i25.3012

16. Hamer OW, Aguirre DA, Casola G, Lavine JE, Woenckhaus M, Sirlin CB. Fatty liver: imaging patterns and pitfalls. Radiographics. (2006) 26:1637-53. doi: $10.1148 / \mathrm{rg} .266065004$

17. Burrows R, Correa-Burrows P, Reyes M, Blanco E, Albala C, Gahagan S. Low muscle mass is associated with cardiometabolic risk regardless of nutritional status in adolescents: a cross-sectional study in a chilean birth cohort. Pediatr Diabetes. (2017) 18:895-902. doi: 10.1111/pedi.12505

18. Kim S, Valdez R. Metabolic risk factors in U.S. youth with low relative muscle mass. Obes Res Clin Pract. (2015) 9:125-32. doi: 10.1016/j.orcp.2014.05.002

19. Kim K, Hong S, Kim EY. Reference values of skeletal muscle mass for Korean children and adolescents using data from the Korean National Health and Nutrition Examination Survey 2009-2011. PLoS ONE. (2016) 11:e0153383. doi: 10.1371/journal.pone.0153383

20. Kleiner DE, Brunt EM, Van Natta M, Behling C, Contos MJ,Cummings OW, et al. Design and validation of a histological scoring system for nonalcoholic fatty liver disease. Hepatology. (2005) 41:1313-21. doi: 10.1002/hep.20701

21. Brunt EM, Kleiner DE, Wilson LA, Belt P, Neuschwander-Tetri BA;NASH Clinical Research Network (CRN). Nonalcoholic fatty liver disease (NAFLD) activity score and the histopathologic diagnosis in NAFLD: distinct clinicopathologic meanings. Hepatology. (2011) 53:810-20. doi: 10.1002/hep. 24127

22. National High Blood Pressure Education Program Working Group on High Blood Pressure in Children and Adolescents. The fourth report on the diagnosis, evaluation, and treatment of high blood pressure in children and adolescents. Pediatrics. (2004) 114(2 Suppl. 4th Report):S555-76.

23. Cook S, Auinger P, Huang TT. Growth curves for cardio-metabolic risk factors in children and adolescents. J Pediatr. (2009) 155:S6.e15-S6.e26. doi: 10.1016/j.jpeds.2009.04.051

24. Shashaj B, Luciano R, Contoli B, Morino GS, Spreghini MR, Rustico C, et al. Reference ranges of hOMA-IR in normal-weight and obese young Caucasians. Acta Diabetol. (2016) 53:251-60. doi: 10.1007/s00592-015-0782-4

25. Lee YH, Jung KS, Kim SU, Yoon HJ, Yun YJ, Lee BW, et al. Sarcopaenia is associated with NAFLD independently of obesity and insulin resistance: nationwide surveys (KNHANES 2008-2011). J Hepatol. (2015) 63:486-93. doi: 10.1016/j.jhep.2015.02.051

26. Petta S, Ciminnisi S, Di Marco V, Cabibi D, Cammà C, Licata A, et al. Sarcopenia is associated with severe liver fibrosis in patients with non-alcoholic fatty liver disease. Aliment Pharmacol Ther. (2017) 45:510-8. doi: 10.1111/apt.13889

27. Lee YH, Kim SU, Song K, Park JY, Kim DY, Ahn SH, et al. Sarcopenia is associated with significant liver fibrosis independently of obesity and insulin 
resistance in nonalcoholic fatty liver disease: nationwide surveys (KNHANES 2008-2011). Hepatology. (2016) 63:776-86. doi: 10.1002/hep.28376

28. Hong HC, Hwang SY, Choi HY, Yoo HJ, Seo JA, Kim SG, et al. Relationship between sarcopenia and nonalcoholic fatty liver disease: the Korean sarcopenic obesity study. Hepatology. (2014) 59:1772-8. doi: 10.1002/hep.26716

29. Koo BK, Kim D, Joo SK, Kim JH, Chang MS, Kim BG, et al. Sarcopenia is an independent risk factor for non-alcoholic steatohepatitis and significant fibrosis. J Hepatol. (2017) 66:123-31. doi: 10.1016/j.jhep.2016.08.019

30. Kim G, Lee SE, Lee YB, Jun JE, Ahn J, Bae JC, et al. Relationship between relative skeletal muscle mass and nonalcoholic fatty liver disease: a 7-year longitudinal study. Hepatology. (2018) 68:1755-68. doi: 10.1002/hep.30049

31. Forbes GB. Lean body mass and fat in obese children. Pediatrics/ (1964) 34:308-14.

32. Peterson MD, Zhang P, Saltarelli WA, Visich PS, Gordon PM. Low muscle strength thresholds for the detection of cardiometabolic risk in adolescents. Am J Prev Med. (2016) 50:593-9. doi: 10.1016/j.amepre.2015.09.019

33. Kim JH, Park YS. Low muscle mass is associated with metabolic syndrome in Korean adolescents: the Korea national health and nutrition examination survey 2009-2011. Nutr Res. (2016) 36:1423-8. doi: 10.1016/j.nutres.2016.09.013

34. Murphy MJ, Metcalf BS, Jeffery AN, Voss LD, Wilkin TJ. Does lean rather than fat mass provide the link between birth weight, BMI, and metabolic risk? Early Bird 23. Pediatr Diabetes. (2006) 7:211-4. doi: 10.1111/j.1399-5448.2006.00180.x

35. Blakeley CE, Van Rompay MI, Schultz NS, Sacheck JM. Relationship between muscle strength and dyslipidemia, serum 25(OH)D, and weight status among diverse schoolchildren: a cross-sectional analysis. BMC Pediatr. (2018) 18:23. doi: 10.1186/s12887-018-0998-x

36. Heymsfield SB, Gonzalez MC, Lu J, Jia G, Zheng J. Skeletal muscle mass and quality: evolution of modern measurement concepts in the context of sarcopenia. Proc Nutr Soc. (2015) 74:355-66. doi: $10.1017 /$ S0029665115000129

37. Nachit M, Leclercq IA. Emerging awareness on the importance of skeletal muscle in liver diseases: time to dig deeper into mechanisms! Clin Sci. (Lond). (2019) 133:465-81. doi: 10.1042/CS20180421

38. Orsso CE, Tibaes JRB, Oliveira CLP, Rubin DA, Field CJ, Heymsfield SB, et al. Low muscle mass and strength in pediatrics patients: why should we care? Clin Nutr. (2019) 38:2002-15. doi: 10.1016/j.clnu.2019.04.012

39. Eslamparast T, Montano-Loza AJ, Raman M, Tandon P. Sarcopenic obesity in cirrhosis-The confluence of 2 prognostic titans. Liver Int. (2018) 38:1706-17. doi: 10.1111/liv.13876

40. Stenholm S, Alley D, Bandinelli S, Griswold ME, Koskinen S, Rantanen T, et al. The effect of obesity combined with low muscle strength on decline in mobility in older persons: results from the inCHIANTI study. Int J Obes. (Lond). (2009) 33:635-44. doi: 10.1038/ijo.2009.62

41. Van Aller C, Lara J, Stephan BCM, Donini LM, Heymsfield S, Katzmarzyk PT, et al. Sarcopenic obesity and overall mortality: results from the application of novel models of body composition phenotypes to the national health and nutrition examination survey 1999-2004. Clin Nutr. (2019) 38:264-70. doi: 10.1016/j.clnu.2018.01.022

42. Buckinx F, Landi F, Cesari M, Fielding RA, Visser M, Engelke K, et al. Pitfalls in the measurement of muscle mass: a need for a reference standard. J Cachexia Sarcopenia Muscle. (2018) 9:269-78. doi: 10.1002/jcsm.12268

43. Mangus RS, Bush WJ, Miller C, Kubal CA. Severe sarcopenia and increased fat stores in pediatric patients with liver, kidney, or intestine failure. J Pediatr Gastroenterol Nutr. (2017) 65:579-83. doi: 10.1097/MPG.0000000000001651

44. Lurz E, Patel H, Frimpong RG, Ricciuto A, Kehar M, Wales PW, et al. Sarcopenia in children with end-stage liver disease. J Pediatr Gastroenterol Nutr. (2018) 66:222-6. doi: 10.1097/MPG.0000000000001792

45. Zamboni M, Gattazzo S, Rossi AP. Myosteatosis: a relevant, yet poorly explored element of sarcopenia. Eur Geriatr Med. (2019) 10:5-6. doi: 10.1007/s41999-018-0134-3

46. Park BS, Yoon JS. Relative skeletal muscle mass is associated with development of metabolic syndrome. Diabetes Metab J. (2013) 37:458-64. doi: $10.4093 / \mathrm{dmj} .2013 .37 .6 .458$
47. McCarthy HD, Samani-Radia D, Jebb SA, Prentice AM. Skeletal muscle mass reference curves for children and adolescents. Pediatr Obes. (2014) 9:249-59. doi: 10.1111/j.2047-6310.2013.00168.x

48. Vos MB, Abrams SH, Barlow SE, Caprio S, Daniels SR, Kohli R, et al. NASPGHAN clinical practice guideline for the diagnosis and treatment of nonalcoholic fatty liver disease in children: recommendations from the expert committee on NAFLD (ECON) and the north American society of pediatric gastroenterology, hepatology and nutrition (NASPGHAN). J Pediatr Gastroenterol Nutr. (2017) 64:319-34. doi: 10.1097/MPG.0000000000001482

49. Ezaizi Y, Kabbany MN, Conjeevaram Selvakumar PK, Sarmini MT, Singh A, Lopez R, et al. Comparison between non-alcoholic fatty liver disease screening guidelines in children and adolescents. JHEP Rep. (2019) 1:259-64. doi: 10.1016/j.jhepr.2019.06.005

50. Bhanji RA, Narayanan P, Allen AM, Malhi H, Watt KD. Sarcopenia in hiding: The risk and consequence of underestimating muscle dysfunction in nonalcoholic steatohepatitis. Hepatology. (2017) 66:2055-65. doi: 10.1002/hep.29420

51. Klip A, Pâquet MR. Glucose transport and glucose transporters in muscle and their metabolic regulation. Diabetes Care. (1990) 13:228-43. doi: 10.2337/diacare.13.3.228

52. Petersen KF, Dufour S, Savage DB, Bilz S, Solomon G, Yonemitsu S, et al. The role of skeletal muscle insulin resistance in the pathogenesis of metabolic syndrome. PNAS. (2007) 104:12587-94 doi: 10.1073/pnas.0705408104

53. Beyer I, Mets T, Bautmans I. Chronic low-grade inflammation and agerelated sarcopenia. Curr Opin Clin Nutr Metab Care. (2012) 15:12-22. doi: 10.1097/MCO.0b013e32834dd297

54. Pratesi A, Tarantini F, Di Bari M. Skeletal muscle: an endocrine organ. Clin Cases Miner Bone Metab. (2013) 10:11-4. doi: 10.11138/ccmbm/2013.10.1.011

55. Pedersen BK. The diseasome of physical inactivity-and the role of myokines in muscle-fat cross talk. J Physiol. (2009) 587(Pt 23):5559-68. doi: 10.1113/jphysiol.2009.179515

56. Johnson NA, George J. Fitness versus fatness: moving beyond weight loss in nonalcoholic fatty liver disease. Hepatology. (2010) 52:370-81. doi: 10.1002/hep.23711

57. Keating SE, Hackett DA, George J, Johnson NA. Exercise and non-alcoholic fatty liver disease: a systematic review and meta-analysis. J Hepatol. (2012) 57:157-66. doi: 10.1016/j.jhep.2012.02.02358

58. Mandato C, Di Nuzzi A, Vajro P. Nutrition and liver disease. Nutrients. (2017) 10:E9. doi: 10.3390/nu10010009

59. Hammad A, Kaido T, Aliyev V, Mandato C, Uemoto S. Nutritional therapy in liver transplantation. Nutrients. (2017) 9:E1126. doi: 10.3390/nu9101126

60. Trouwborst I, Verreijen A, Memelink R, Massanet P, Boirie Y, Weijs P, et al. Exercise and nutrition strategies to counteract sarcopenic obesity. Nutrients. (2018) 10:E605. doi: 10.3390/nu10050605

61. Hsu KJ, Liao CD, Tsai MW, Chen CN. Effects of exercise and nutritional intervention on body composition, metabolic health, and physical performance in adults with sarcopenic obesity: a meta-analysis. Nutrients. (2019) 11:E2163. doi: 10.3390/nu11092163

62. Vajro P, Mandato C, Veropalumbo C, De Micco I. Probiotics: a possible role in treatment of adult and pediatric non alcoholic fatty liver disease. Ann Hepatol. (2013) 12:161-3. doi: 10.1016/S1665-2681(19)31401-2

63. Ticinesi A, Nouvenne A, Cerundolo N, Catania P, Prati B, Tana C, et al. Gut microbiota, muscle mass and function in aging: a focus on physical frailty and sarcopenia. Nutrients. (2019) 11:E1633. doi: 10.3390/nu11071633

Conflict of Interest: The authors declare that the research was conducted in the absence of any commercial or financial relationships that could be construed as a potential conflict of interest.

Copyright (๑) 2020 Pacifico, Perla, Andreoli, Grieco, Pierimarchi and Chiesa. This is an open-access article distributed under the terms of the Creative Commons Attribution License (CC BY). The use, distribution or reproduction in other forums is permitted, provided the original author(s) and the copyright owner(s) are credited and that the original publication in this journal is cited, in accordance with accepted academic practice. No use, distribution or reproduction is permitted which does not comply with these terms. 